\title{
THE MANAGEMENT OF PATIENTS WITH POLYPOSIS OF THE SMALL INTESTINE
}

By David Bailey, M.Chir., F.R.C.S. Surgeon, Barnet General Hospital and Assistant, Surgical Unit, University College Hospital

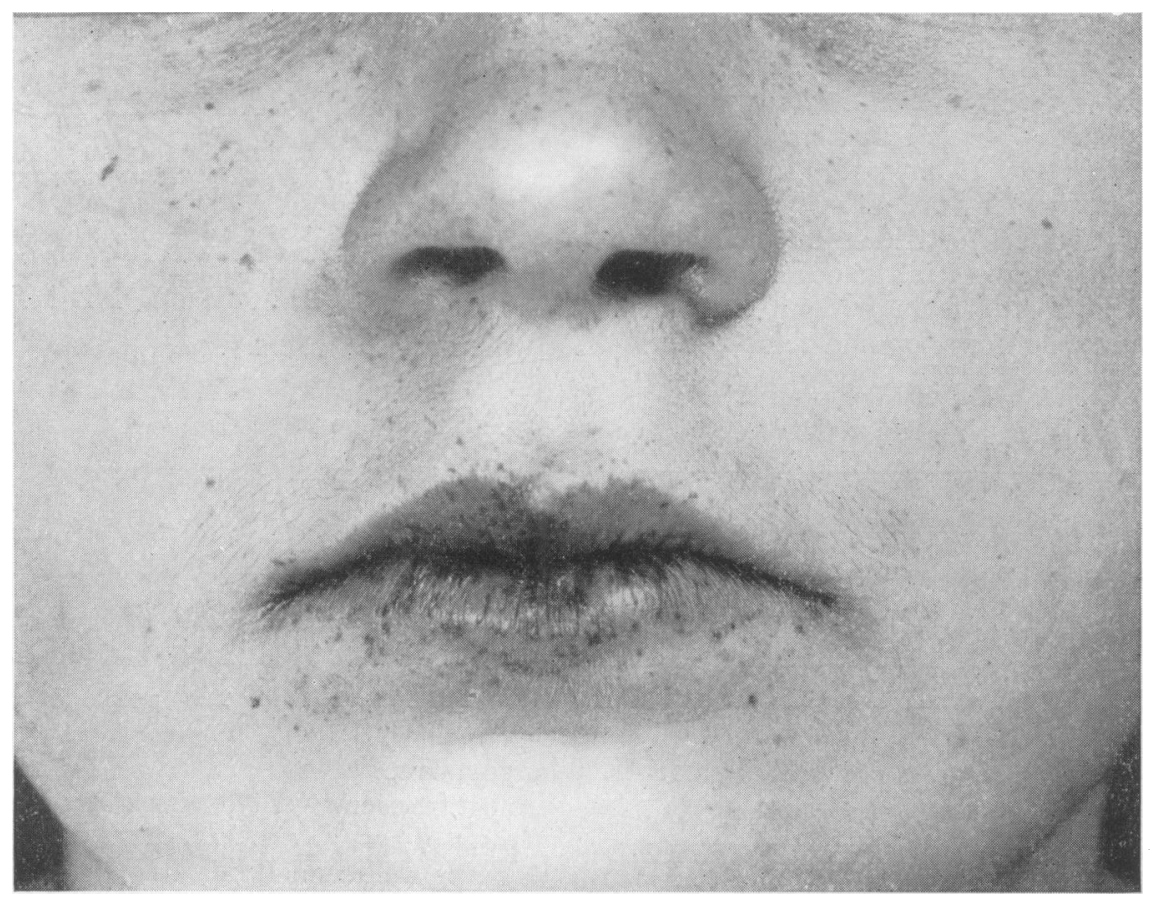

FIG. I.

\section{Introduction}

Benign adenomas are very much more uncommon in the small intestine than in the colon, but when they do occur they are frequently multiple, and the condition is then known as polyposis of the small intestine. Any surgeon is likely to meet with only a few examples of this condition during the course of his career, and, probably for this reason, patients with polyposis of the small intestine often pose difficult problems in diagnosis and treatment. Thanks to the work of Dr. Cuthbert Dukes and the surgeons of St. Mark's Hospital, the treatment of colonic polyposis may now be said to be almost standardized (Dukes, I952a; Thompson, I958) but the treatment of the rarer small intestinal tumours remains more controversial. It is only recently that the collection of several published cases has made it possible to formulate some plan of campaign in the management of this disease.

In the last ten years, interest has been stimulated by the wider recognition of a syndrome in which polyposis of the gastro-intestinal tract, with involvement of the small intestine in all cases, is associated with a peculiar melanin pigmentation of the lips, face and oral mucosa (Fig. I). This association is usually known as the Peutz syndrome, after the Dutchman who originally described it in I92 I (Peutz, I92I). Credit for its general recognition must be given to Jeghers of America who, in 
I948, provided a classical review of the syndrome with a description of several new cases (Jeghers, McKusick and Katz, I949). Since I948 several further cases have been reported, and review of the literature in 1957 yielded 80 authentic examples (Bailey, 1957). Dormandy (1957) has recently given a very complete description of the disease. However, although recognition of the Peutz syndrome has served to call attention to the problem of small intestinal polyposis, it must not be thought that these tumours do not occur in the small intestine in the absence of muco-cutaneous pigmentation; in many cases multiple polyps are present in the jejunum and ileum without any of the tell-tale melanin spots of the lips and face.

\section{The Diagnosis of Polyposis}

Rational treatment is based on complete diagnosis and for that reason a few words on the symptomatology and diagnostic difficulties will not be out of place here. If muco-cutaneous pigmentation is present recognition of the cause of obscure intestinal symptoms should not be long delayed. 'It is nice to find a condition which one can diagnose from across the ward' comments Ravitch (1955). If, however, the pigmentation is absent, passes unnoticed, or its significance is not recognized, then the diagnosis of small intestinal polyposis may prove very difficult and may only be made at laparotomy.

The symptoms produced by these tumours fall into three groups (Olson, Dockerty and Gray, I95I):

I. Symptoms due to obstruction.

2. Bleeding.

3. Symptoms produced by local irritability of the intestine.

The first symptoms most often appear between the ages of 20 and 30 years, although many younger and older patients have been reported. Obstruction, usually due to recurrent intussusception, causes attacks of intermittent abdominal pain, often with vomiting. Bleeding may result in tarry stools or the passage of dark red blood, according to the site of the tumours but, in some cases, the loss of blood may be occult, showing itself in the guise of an unexplained iron-deficiency anaemia. Local irritability may cause intestinal hurry, resulting in chronic diarrhoea and various deficiency syndromes; duodenal tumours may simulate peptic ulcer or even carcinoma of the stomach. Clinical awareness is the most important step towards arriving at the diagnosis: laboratory investigations are unlikely to be of great help in this direction.

\section{The Aims of Treatment}

Surgical treatment is required to rid the patient of as many polyps as possible in order to avoid the

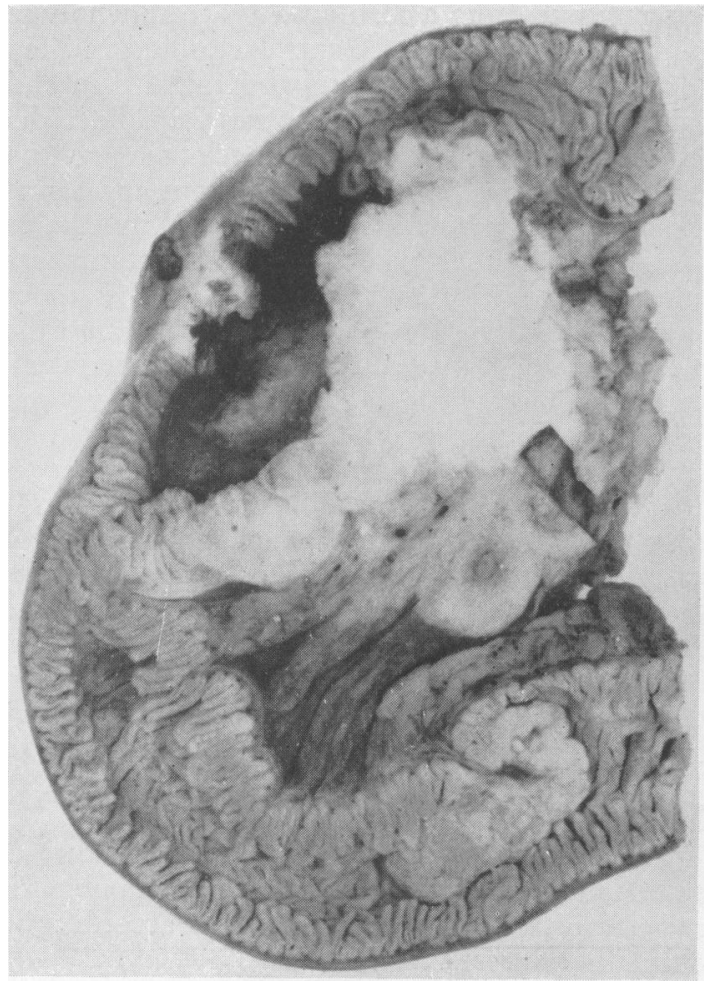

FIG. 2.

dangerous complication of intussusception, to reduce blood loss, and to prevent malignant degeneration. The occurrence of malignant degeneration is now accepted in the case of familial polyposis of the colon, in which disease cancer is most frequently diagnosed about i 5 years after the onset of symptoms (Dukes, I952). I believe that polyps of the small intestine are liable to the same risk of carcinomatous change. In a recent review of 67 published examples of the Peutz syndrome, the occurrence of carcinoma of the small intestine (diagnosed histologically) was noted in 13 -an incidence of about I9 per cent. (Bailey, 1957). It must also be remembered that in the past many of these patients have met with death at a young age from some complication such as intussusception. With a lowered mortality we may meet with a higher incidence of carcinoma in the future. Dormandy (1957) challenges this view, stating that in reported cases the diagnosis of malignant disease has been made on the evidence of the microscope and has not been borne out by the subsequent progress of the patient. That the risk is real, however, is suggested by the specimen shown in Fig. 2.

The specimen consists of $2 \mathrm{ft}$. of jejunum. It 
was removed from a man aged 69 years who was admitted to the Barnet General Hospital in May I958, complaining of central abdominal pain and flatulence after meals. A mass was palpable in the left hypochondrium and a carcinoma of the stomach was suspected; but barium meal was negative. No pigmentation of the oral mucosa or face was present in this patient. Laparotomy was undertaken and a large carcinoma of the upper jejunum was found which had ulcerated into the mesentery. A palliative resection was undertaken. In the photograph the large ulcerated tumour can be seen in the proximal part of the intestine, in the more distal part a large polypoid tumour is apparent. There were also a few smaller polyps which are not obvious in the photograph. Microscopy of the ulcerated tumour showed a mucus secreting adenocarcinoma; the large polyp shows the histological appearances of a benign adenoma. Although there is no proof that the carcinoma developed from a previously existing benign polyp, it appears probable that this may have occurred.

\section{The Nature of Treatment}

For the purposes of deciding on the nature of treatment, these cases of small intestinal polyposis may be divided into several groups according to the mode of presentation.

\section{Patients Presenting as Emergencies, with Intestinal Obstruction}

Although intussusception in these cases tends to be chronic, and less dramatic than the more common infantile variety, this complication may sometimes demand urgent surgical intervention; to be undertaken as soon as dehydration and electrolyte imbalance have been corrected.

The cause of the obstruction may be diagnosed on clinical grounds, particularly if the Peutz pigmentation is present and recognized. If this is the case, a short period of conservative treatment is worth while. This may be effective, permitting postponement of surgery so that a planned operation can be undertaken under more favourable circumstances. The main-stays of conservative treatment are gastro-intestinal suction and the administration of parenteral fluids. Giving the patient an enema may abort the attack and should be tried.

On the other hand, intussusception may be found unexpectedly on laparotomy, and the extent of the surgical procedure will then depend on the condition of the patient and the experience of the surgeon. The intussusception must be reduced if possible; the causative tumour will then be discovered and should be removed by enterotomy or resection of a short length of intestine. It is unsafe to leave the tumour, as, if this is done, early recurrence of the obstruction is likely. After the polyp has been dealt with, the rest of the intestine should be examined for the presence of further polyps if the patient's general condition permits; any large tumours so discovered should be removed. In many cases, however, the patient is too ill to allow this further exploration and surgical treatment should then be confined to dealing with the intussusception and its cause. This will usually be the case when the intussusception proves to be irreducible and has to be resected-if the patient is in extremis the subsequent anastomosis may be omitted; after resection the two ends of intestine may be brought out as a double-barrelled enterostomy to be joined up at a later date.

\section{Patients Presenting as Emergencies, with Haemorrhage}

Haemorrhage from polyps of the small bowel is usually episodic and may not require any medical aid. On occasions, however, it may be more severe and the patient may present in a state of oligaemic shock. Treatment should in the first place be conservative. That is to say, the patient is admitted to hospital and treated in much the same way as if he were suffering from a bleeding peptic ulcer. Morphia is given, preferably intrao venously, and nothing is allowed by mouth. A estimate should be made of the volume of bloo $\Phi$ lost and this amount is replaced intravenously at $\vec{a}$ rapid rate. Blood transfusion is continued slowly until bleeding stops, as it usually does after an hour or two. If blood loss continues, or is taking place faster than it can be replaced, an immediate laparotomy will offer the only chance of saving life. After the abdomen is opened the samll intestine is rapidly palpated and portions of intestine containing any large tumours are isolated by applying occlusion clamps. This will stop the bleeding and the tumours can be dealt with by enterotomy or resection after the patient's condition has been restored. The difficulty about surgical treatment of this complication is to be sure which polyps are responsible for the haemorrhage. Fortunately bleeding of this catastrophic type is rare-conservative methods will, as a rule, tide the patient over and the tumours can be dealt with at comparative leisure as described below.

\section{Patients Presenting with more Chronic Symptoms}

Time is of less importance when dealing with these patients. Planned surgical treatment can be undertaken after a period of medical preparation $\Phi$ designed to correct nutritional deficiencies and to fit the patient for operation.

A general medical examination will reveal any serious intercurrent disease and a chest X-ray will 


\section{Recent Trends in Chronic Bronchitis} Edited by NEVILLE C. OSWALD M.D.(Cantab.), F.R.C.P.(Lond.)

Physician, St. Bartholomew's and Brompton Hospitals, London; Honorary Physician to H.M. The Queen

\section{Introduction to Surgery}

Edited by D. H. PATEY

M.S.(Lond.), F.R.C.S.(Eng.)

Surgeon, The Middlesex Hospital;

Director of Surgical Studies, The Middlesex Hospital Medical School

\author{
General Pathology \\ (2nd edition) \\ Edited by SIR HOWARD FLOREY \\ M.D., F.R.C.P., F.R.S. \\ Professor of Pathology, University of Oxford
}

viii $+200 p p$.

76 illustrations,

2 colour plates

(1958) 30s. net

$x i i+228 p p$.

54 illustrations

(1958) Clothbound

17s. 6d. net

Paper-covered

9s. 6d. net

$x v i+932 p p$.

410 illustrations,

3 colour plates

(1958) 84s. net

$v i i i+140 p p$.

42 illustrations

(1957) 20s. net
Assistant Director, Department of Surgical Studies, Middlesex Hospital

by L. P. LE QUESNE

(2nd edition)

D.M.(Oxon), F.R.C.S.(Eng.)

Newman Street, London, W. be needed in every case. Sigmoidoscopy should be carried out as a routine, as, in about half of the recorded examples of small intestine polyposis, tumours have been present in the colon and rectum as well. A barium enema should be performed for the same reason. Barium meal and follow-through examination may demonstrate the presence of polyps in the duodenum or stomach (Tanner, 195I), but gastroscopy is only occasionally performed. Laboratory investigations should include estimation of the haemoglobin, serum proteins, prothrombin concentration, serum electrolytes and blood group.

Medical Treatment. This is only a preliminary to surgical treatment and cannot influence the progress of the disease. Iron deficiency must be corrected-if slight, by the oral administration of a preparation such as Tabs. Ferrous Gluconate (mg. 300) I-2 t.d.s., or, if more severe, by intramuscular injection of 'Imferon' ( 2 c.c. daily). Only if anaemia is very severe or time is short, should pre-operative blood transfusion be necessary. Intestinal hurry may be responsible for various vitamin deficiencies and vitamin supplements should be given (e.g. Tabs. vitaminorum). If the prothrombin concentration is low, vitamin $\mathrm{K}$.I. is given parenterally.

Definitive surgical treatment. Before surgery is undertaken the bowel contents should be sterilized as far as possible by antibiotics. The usual plan is to give succinyl sulphathiazole for five days before operation (in doses of $3 \mathrm{~g}$. every six hours). On the day previous to operation oral streptomycin is given in addition ( $5 \mathrm{~g}$. in roo c.c. water, b.d.) and a final dose is given on the morning of operation.

The exact nature of surgical treatment will depend on the distribution of the polyps. If the colon is extensively involved this should usually be treated first, along the lines laid down for treatment of familial polyposis of the colon, the rectum being conserved if at all feasible. It may be mentioned in passing that it is important that polyposis confined to the colon should not be confused with the rarer polyposis involving the whole gastrointestinal tract, including the small intestine. A surgeon is indeed in a dilemma if he has resected the whole colon and rectum and, having overlooked the presence of polyps in the jejunum or ileum, is surprised to find that bleeding continues from the ileostomy stoma.

When treating polyposis of the small intestine the most important consideration is to bear in mind the need to conserve as much of the absorptive part of the alimentary tract as is possible. Examination of the whole intestine is essential and 
a long exploratory incision is necessary; a transverse incision across both recti just below the level of the umbilicus or a long right paramedian incision are both adequate. The whole alimentary tract should be carefully palpated before any attempt is made to remove the tumours. In a few cases the polyps are localized to short lengths of intestine which may be resected, although it must be realized that small tumours can easily be missed on palpation of the unopened intestine. If the small intestinal polyposis is more generalized resective surgery is contra-indicated as this may result in jejuno-ileal insufficiency. Probably the most effective way of dealing with the problem is by the method of serial enterotomy described by Vary (1956). The jejunum is opened by a $1 \frac{1}{2}$-in. longitudinal incision through the anti-mesenteric border, about ro in. below its commencement. Tissue forceps are passed upwards through the opening and made to grip the mesenteric border as high as possible. Partly by traction on the forceps and partly by pressure from without, the jejunum is intussuscepted downwards and made to protrude through the enterotomy. The mucous surface is then facing outwards and is available for inspection and removal of any tumours. The 'intussusception' is then reduced and the intestine below is delivered upwards in similar fashion and cleared of polyps in turn. The enterotomy is then closed transversely in two layers with continuous cat-gut. A second enterotomy is then made about 20 in. below the first and the procedure is continued seriatim down to the ileocaecal junction.

It is not claimed that this procedure will result in the clearance of all the adenomas, as tiny tumours are easily overlooked, but it at least offers a reasonable chance of ridding the patient of most of the polyps while conserving jejuno-ileal function.

The post-operative course is usually uneventful but a degree of ileus is common for a few days so that gastro-intestinal suction through a Ryle's tube and the administration of parenteral fluids should be continued until intestinal motility returns. The prophylactic administration of intramuscular penicillin and streptomycin is a wise precaution and is normally continued for five days after operation.

\section{The Treatment of the Symptom-free Patient}

The patient who is known to harbour polyps in his small intestine but who has no symptoms presents a difficult problem. It is considered that such a patient should not be treated surgically, but should be kept under careful observation. If $\overrightarrow{\mathrm{O}}$ however, symptoms arise during this period,, surgical treatment is required as these symptoms may herald the development of carcinoma. $\mathrm{\Phi}$ Patients who show the typical muco-cutaneous pigmentation of the Peutz syndrome but who ${ }^{\infty}$ have no symptoms to suggest polyposis are also $\vec{\circ}$ included in this category. As several cases have $\vec{\overrightarrow{ }}$ been recorded of pigmentation without polyposis $\omega$ (Dormandy, 1957) there seems little point in sub-8 jecting these patients to exhaustive investigation; they should be observed carefully until symptoms occur.

\section{The Need for Follow-up}

Dormandy (1957) emphasises that this disease advances by ' segmental spurts,' with long periods of quiescence intervening between spurts of growth. Therefore it is important that all patients? should be most carefully followed-up as, even when the intestine has been cleared of polyps as described above, fresh polyps may arise in the future. Follow-up should include the tracing. and examination of any relatives as, by this means: it may prove possible to add to our knowledge of the inheritance of this disease. The studies of Dukes (1952b) on the inheritance of polyposis of $\gtrsim$ the colon provide a model for such an investigation. $\stackrel{\square}{\stackrel{2}{\Omega}}$

\section{Acknowledgments}

I am indebted to Mr. Derek Ellis, of University College Hospital, for the photograph in Fig. 2.

\section{BIBLIOGRAPHY}

BAILEY, D. (195\%), Brit. med. F., ii, 433.

DORMANDY, T. L. (1957), New Engl. F. Med., 256, 1093, I 51 and 1186 .

DUKES, C. E. (1952a), Ann. roy. Coll. Surg. Engl., 10, 293.

DUKES, C. E. (1952b), Ann. Eugen. (Camb.), 17, I.

JEGHERS, H., MCKUSICK, V. A., and KATZ, K. H. (1949), New Engl. F. Med., 241, 993 and I031.

OLSON, J. D., DOCKERTY, M. B., and GRAY, H. K. (I95I), Ann. Surg., 134, 195.

PEUTZ, J. L. A. (I921), Ned. Maandschr. Geneesk, Iо, I34.

RAVITCH, M. M. (1955), Ann. Surg., 141, 672.

TANNER, N. C. (195I), Proc. roy. Soc. Med., 44, 405.

THOMPSON, H. R. (1958), Proc. roy. Soc. Med., 5I, 241.

VARY, E. P. (1956), Amer. F. Surg., 91, 152. 